\title{
Comparative Analysis of Hyaluronidase-Mediated Degradation Among Seven Hyaluronic Acid Fillers in Hairless Mice
}

This article was published in the following Dove Press journal: Clinical, Cosmetic and Investigational Dermatology

\author{
Seong Sung Kwak $\mathbb{D}^{1,2}$ \\ Kwang Ho Yoon' \\ Jin Hee Kwon' \\ Won-Ho Kang' \\ Chang-Hoon Rhee iD $^{3}$ \\ Gi-Hyeok Yang' \\ Deu John M Cruz (iD) \\ Woo-Chan Son ${ }^{2}$ \\ 'Gwanggyo R\&D Center, Medytox Inc., \\ Suwon-si, Gyeonggi-do, Korea; \\ ${ }^{2}$ Department of Pathology, Asan Medical \\ Center, Seoul, Korea; ${ }^{3}$ Osong R\&D \\ Center, Medytox Inc., Cheongju-si, \\ Chungcheongbuk-do, Korea
}

Purpose: Hyaluronic acid (HA) is the most common injectable dermal filler used for softtissue augmentation, and can be removed non-surgically by directly injecting hyaluronidase. In this study, the hyaluronidase-mediated degradation of different types of HA fillers implanted subcutaneously at the back of hairless mice having filler residence time of four days or three months were compared.

Methods: Two sites at the back of female hairless mice were subcutaneously implanted with $0.1-\mathrm{mL}$ of one of the seven HA fillers (NLL, NL, NDL, NVL, and ND, JUV $\mathrm{X}_{+}$, and RES $_{\text {LYFT }}$ ) and injected with $30 \mathrm{IU}$ or $60 \mathrm{IU}$ hyaluronidase per $0.1-\mathrm{mL}$ filler after reaching a filler residence time of 4 or 91 days, respectively. Filler bolus projection was measured using three-dimensional optical imaging over a $72 \mathrm{~h}$ period, and the implantation sites were histologically examined 2 weeks after hyaluronidase injection.

Results: Following hyaluronidase injection, all seven HA fillers showed a rapid decrease of filler volume within $24 \mathrm{~h}$, and complete degradation was confirmed by histological examination after 2 weeks. There was no significant difference in filler volume reduction rate among the seven HA fillers, and no evidence of macroscopic or microscopic adverse effects were observed at the implantation sites.

Conclusion: All seven HA fillers show comparable susceptibility to hyaluronidasemediated degradation. HA fillers with prolonged filler residence time may require a higher dose of hyaluronidase to achieve efficient degradation owing to tissue integration.

Keywords: soft tissue augmentation, HA filler volume, 3D optical imaging, filler residence time, tissue integration

\section{Introduction}

Hyaluronic acid (HA) is a naturally occurring glycosaminoglycan polymer of disaccharide repeats comprising D-glucuronic acid and N-acetyl-glucosamine, linked via alternating $\beta-1,4$ and $\beta-1,3$ glycosidic bonds. HA is a clear, viscous liquid composed of unbranched single-chain polymers of varying sizes, ranging from $10^{5}$ to $10^{7} \mathrm{kDa}$. HA forms an essential component of connective tissues and organs owing to its abilities of binding and retaining water molecules, keeping the tissues hydrated. ${ }^{1,2} \mathrm{HA}$ is also an acid mucopolysaccharide found in bacterial capsules; a characteristic exploited by industries for the production of non-animal derived natural lubricant since the last century. ${ }^{3} \mathrm{HA}$ is structurally homogenous across different species. Because of its unique physical and mechanical properties as well as biocompatibility, HA is considered as an ideal material for soft tissue augmentation and facial rejuvenation. ${ }^{4,5}$

Deu John M Cruz

Non-Clinical Development Department,

Medytox Gwanggyo R\&D Center,

II 4 Central town-ro, Yeongtong-gu,

Suwon-si, Gyeonggi-do, Republic of Korea

$\mathrm{Tel}+823180658254$

$\mathrm{Fax}+823180658232$

Email deujohn.cruz@medytox.com 
Recently, the frequency of cutaneous augmentation performed using injectable HA fillers has significantly increased. Consequently, reversibility of this process has become equally important for addressing the incidence of patient dissatisfaction caused by undesirable effects, such as unintended or excessive augmentation and Tyndall effect, as well as for resolving occasional incidence of adverse clinical outcomes, including persistent granulomatous changes, necrosis caused by vascular occlusion resulting from arterial injection, unexpected inflammatory response to the accompanying excipients, and other iatrogenic deformities. A simple, minimally invasive procedure that reverses soft-tissue augmentation was developed using hyaluronidase to dissolve superficially or inappropriately placed HA filler. ${ }^{6,7}$

Hyaluronidases are endo- $\mathrm{N}$-acetyl hexosaminidases that catalyze the dissociation and depolymerization of HA polymers through enzymatic cleavage of glycosidic bonds. In humans, six different hyaluronidases expressed from the hyaluronidase-like genes HYAL1, HYAL2, HYAL3, HYAL4, PH20/SPAM1, and the pseudogene HYALP1 have been identified. ${ }^{8}$ Hyal-1 and Hyal-2 are the two major acid-active hyaluronidases found in most tissues that degrade HA by hydrolyzing the $\beta-1,4$ glycosidic linkage between $N$-acetyl-glucosamine and D-glucuronic acid. Hyal-2 is a GPI-anchored hyaluronidase that cleaves CD44-bound HA polymers into $20-\mathrm{kDa}$ products that are subsequently delivered into endolysosomal compartments where they are further degraded into smaller HA oligosaccharides by Hyal-1 as well as $\beta$ exoglycosidase enzymes. ${ }^{9,10}$ Hyaluronidase-mediated degradation is a highly efficient process and is responsible for the short half-life of endogenous HA and its rapid turnover in the body. ${ }^{11}$

Compared with endogenous HA polymers found in the human body, most HA-based dermal fillers are manufactured as crosslinked high-molecular weight HA polymers through chemical modification using crosslinking agents such as divinyl sulfone, p-phenylene bisethyl carbodiimide, 2,7,8-diepoxyoctane and 1.4-butanediol diglycidyl ether (BDDE). Crosslinking of HA polymers generates a three-dimensional network that enhances the viscoelastic properties of HA fillers and increases their durability against rapid degradation caused by constant exposure to endogenous hyaluronidase, free radical oxidation, and mechanical forces. ${ }^{12-14}$ Two of the most popular commercial HA filler products approved by the USFDA for softtissue augmentation are manufactured using BDDE as cross-linker. The Juvéderm family of products (AbbVie/ Allergan) uses their proprietary "Hylacross" and "Vycross" technology to manufacture monophasic HA fillers, whereas the Restylane family of products (Galderma) manufacture their biphasic HA dermal fillers using their proprietary non-animal stabilized HA (NASHA) and more recently, "XpresHAn" technology. ${ }^{15-17}$ Another series of BDDE-crosslinked HA dermal fillers was recently introduced in South Korea. The Neuramis family of products (NEU series, Medytox, Inc.) are monophasic HA dermal fillers manufactured using stabilized HA through purification and enhancement (SHAPE) technology, generating products with varying degree of crosslinking to suit specific clinical indication. ${ }^{18,19}$

The present study investigated the in vivo hyaluronidase-mediated degradation of five HA dermal fillers belonging to the NEU series in hairless mice and compared them with those of two commercially available HA dermal fillers. Degradation of the HA fillers was monitored via three-dimensional optical imaging, wherein the surface projection imposed by the HA filler bolus implanted at the back of the animals was monitored after hyaluronidase injection, and any adverse effect was assessed via gross and histological examination of the implantation sites. The effect of tissue integration on the susceptibility of these HA fillers to hyaluronidasemediated degradation was also taken into account by comparing the HA fillers that have resided at the implantation sites for four days with those that resided for three months (91 days) prior to hyaluronidase treatment.

\section{Materials and Methods}

The study was conducted at the animal facility of Medytox Inc. Gwanggyo R\&D Center in accordance with the Laboratory Animal Act of Korea (Act No. 15278). Prior to study initiation, all procedures involving the use of laboratory animals were approved by the Institutional Animal Care and Use Committee (IACUC) of Medytox Inc. (Approval No. A-2018-010) following the IACUC guidelines from the Ministry of Food and Drug Safety, Republic of Korea (Registration No. 11-1543061-000268-14).

\section{Animals}

Six to seven-week-old female hairless mice (SKH1- $\mathrm{Hr}^{\text {hr }}$ ) were purchased from Orient Bio, Inc. (Seongnam-si, Korea) and housed in groups of three. The animals were kept in an environment with a 12-h light/dark cycle, 
controlled temperature $\left(23 \pm 3^{\circ} \mathrm{C}\right)$, relative humidity $(55 \pm$ $15 \%)$ and were given free access to food and water.

\section{Hyaluronic Acid Fillers and Study Design}

Five different HA fillers belonging to the NEU series, namely Neuramis Light Lidocaine (NLL), Neuramis Lidocaine (NL), Neuramis Deep Lidocaine (NDL), Neuramis Volume Lidocaine (NVL), and Neuramis Deep (ND), as well as two other commercial HA fillers Juvéderm Ultra Plus XC ( $\left.\mathrm{JUV}_{\mathrm{X}+}\right)$ and Restylane LYFT with Lidocaine $\left(\right.$ RES $_{\text {LYFT }}$ ) were evaluated for their susceptibility to in vivo hyaluronidase-mediated degradation under two different filler residence times: 4 days and 91 days. The commercially available ovine hyaluronidase, LIPORASE (DaeHan New Pharm Co., Ltd., Korea), was used for this study.

Sixty-five mice were segregated into two sets corresponding to the 4- and 91-day filler residence times. Each HA filler was assigned five mice from each set, except NLL, which was assigned five mice from the 4-day filler residence time set only. On day 0 , the animals were given an intraperitoneal dose of $100 \mathrm{mg} / \mathrm{kg}$ ketamine $+10 \mathrm{mg} / \mathrm{kg}$ xylazine prior to filler implantation. The corresponding HA filler $(0.1 \mathrm{~mL})$ was subcutaneously injected at two sites on the back of each animal, namely one caudal and one rostral, forming a projecting bolus at the implantation site (Figure 1A).

On the day corresponding to their designated filler residence time, two animals from each group were euthanized, and the implantation sites were excised for histological examination. The remaining animals were placed under parenteral anesthesia and subsequently injected with $30 \mathrm{IU}$ hyaluronidase directly to the center of the projecting HA filler bolus. Within $30 \mathrm{~min}$ after the first injection, a second injection of 30 IU hyaluronidase was administered on the HA filler implants in the 91-day filler residence time set. HA filler volume at $0,1,6,24,48$, and $72 \mathrm{~h}$ after hyaluronidase injection was evaluated by surface projection measurements using PRIMOS Lite imaging device (CANFIELD Scientific Inc., NJ, USA). Reduction in HA filler volume was reported as percentage change relative to the baseline HA filler volume measured at $0 \mathrm{~h}$ (before hyaluronidase injection). Two weeks after hyaluronidase injection, all animals were euthanized, and implantation sites were excised for histological examining and confirming the degradation of HA fillers.
A

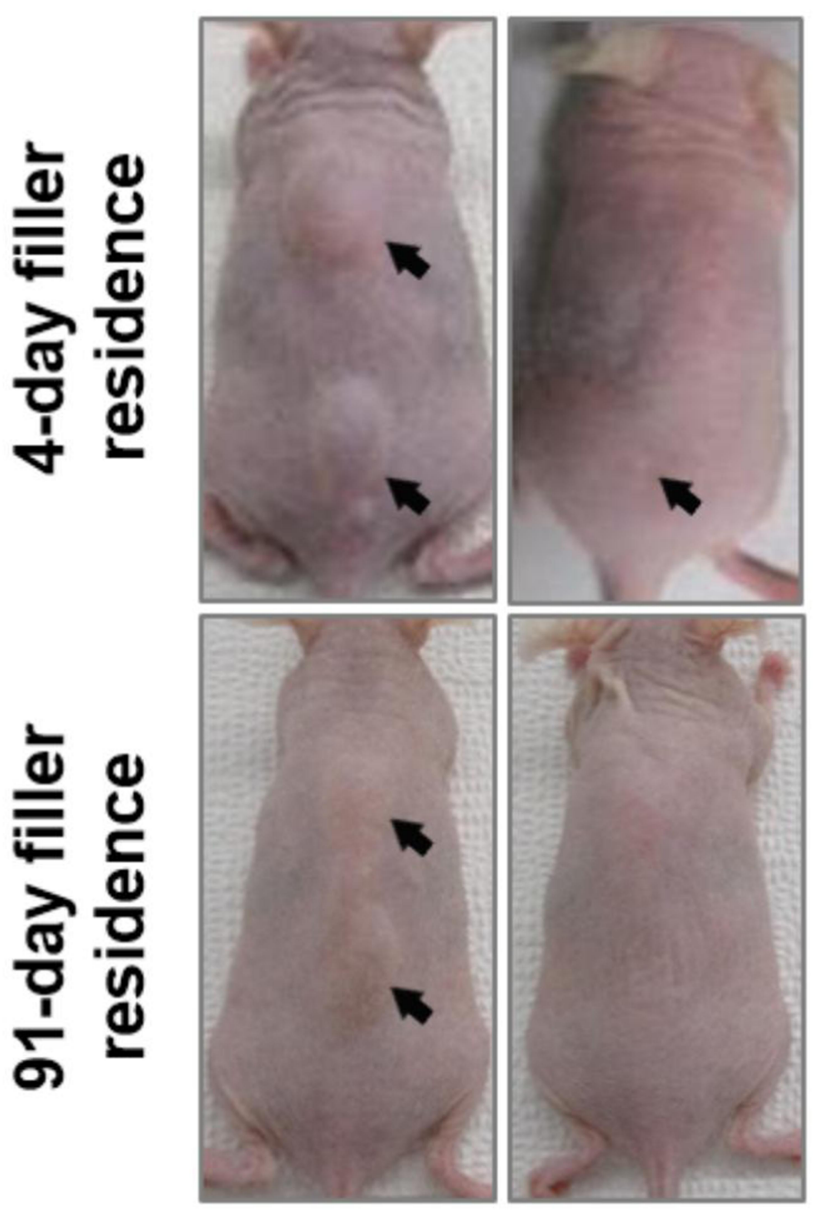

Figure I Hyaluronidase-mediated degradation of subcutaneously implanted HA filler. Hairless mice implanted subcutaneously with $0.1 \mathrm{~mL} \mathrm{NDL}$. Top and bottom panels show NDL with 4- and 91-day filler residence time, respectively, before (A) and $\mathrm{I} \mathrm{h}$ after hyaluronidase injection (B). Arrows mark the implantation sites showing the surface projection of the HA filler bolus.

\section{Histological Evaluation}

Tissue samples excised from the implantation sites were fixed in $10 \%$ formalin solution and embedded in paraffin. Five-micrometer thin sections were stained with hematoxylin and eosin for evaluating tissue histology as well as with Alcian Blue for detecting the embedded HA filler. Local adverse effects of hyaluronidase on the surrounding tissues were assessed by assigning scores to the histological features using a semi-quantitative evaluation system described in ISO $10993-6 .{ }^{20}$ The average histology score was used to determine hyaluronidase treatment either as a nonirritant (0-2.9), slight irritant (3-8.9), moderate irritant (9-15), or severe irritant $(\geq 15.1)$. 


\section{Statistical Analysis}

The average filler volume of the seven types of HA dermal filler implants measured at different time points are presented as percentages relative to their initial volume before hyaluronidase injection. Changes in the mean filler volumes over time were compared using non-parametric methods (Mann-Whitney test) by employing SPSS software (version 25.0, SPSS Inc., IL, USA). $P$ value of $<0.05$ was considered statistically significant.

\section{Results}

\section{Hyaluronidase-Mediated Degradation of Implanted HA Fillers}

Loss of HA filler surface projection is directly proportional to volume reduction of the filler implant and is mainly attributed to hyaluronidase-mediated degradation (Figure 1). For the HA filler implants having a 4-day filler residence time, a single injection of 30 IU hyaluronidase $/ 0.1-\mathrm{mL}$ filler implant showed a significant reduction in HA filler volume within $1 \mathrm{~h}$ after hyaluronidase injection $(p<0.05)$. Complete loss of surface projection was observed from all implantation sites of NLL, NL, and $\mathrm{JUV}_{\mathrm{X}+}$ within $1 \mathrm{~h}$ of hyaluronidase injection, while all implantation sites of NL, NVL, and RES $_{\mathrm{LYFT}}$ showed a complete loss of surface projection within $6 \mathrm{~h}$ of hyaluronidase injection. For NDL and ND, five of the six implantation sites showed a complete loss of surface projection within $6 \mathrm{~h}$ of hyaluronidase injection, with the remaining implantation sites of both HA fillers showing a $96.9 \%$ and $96.8 \%$ reduction in surface projection 72 $\mathrm{h}$ after hyaluronidase injection, respectively (Figure 2A).
For the HA filler implants having a 91-day filler residence time, a second injection of 30 IU hyaluronidase/ $0.1-\mathrm{mL}$ filler implant was administered within $30 \mathrm{~min}$ after the initial injection upon observing a slower rate of filler volume reduction compared with their 4-day filler residence time counterparts (data not shown). A significant reduction in filler volume was observed 1 hour following the administration of hyaluronidase with a total dosage of $60 \mathrm{IU} / 0.1-\mathrm{mL}$ filler implant $(p<0.05)$. All implantation sites showed complete loss of surface projection within 24 $\mathrm{h}$ of hyaluronidase treatment (Figure 2B). In particular, implantation sites of NL and NDL showed $100 \%$ loss of surface projection within $1 \mathrm{~h}$; ND and RES $\mathrm{LYFT}_{\text {, within } 6}$ $\mathrm{h}$; and NVL and $\mathrm{JUV}_{\mathrm{X}+}$ within $24 \mathrm{~h}$ after hyaluronidase injection. Evaluation of hyaluronidase-mediated degradation of NLL having a 91-day filler residence time was not performed after a preliminary study revealed a significant decrease in filler volume occurring at $<90$ days after subcutaneous implantation (data not shown).

\section{Local Effects of Hyaluronidase Treatment}

Following the ISO 10993-6 guidelines for testing the local effects after implantation, histological evaluation of the tissues at the implantation sites conducted at 2 weeks after hyaluronidase injection revealed a mean histology score of $\leq 1.7$ for all HA fillers belonging to the 4-day filler residence time set, and a mean histology score of $\leq 2.4$ for all HA fillers belonging to the 91-day filler residence time set (Table 1). Histological examination of the implantation sites revealed a nearly complete elimination of the HA filler implants having a 4-day filler

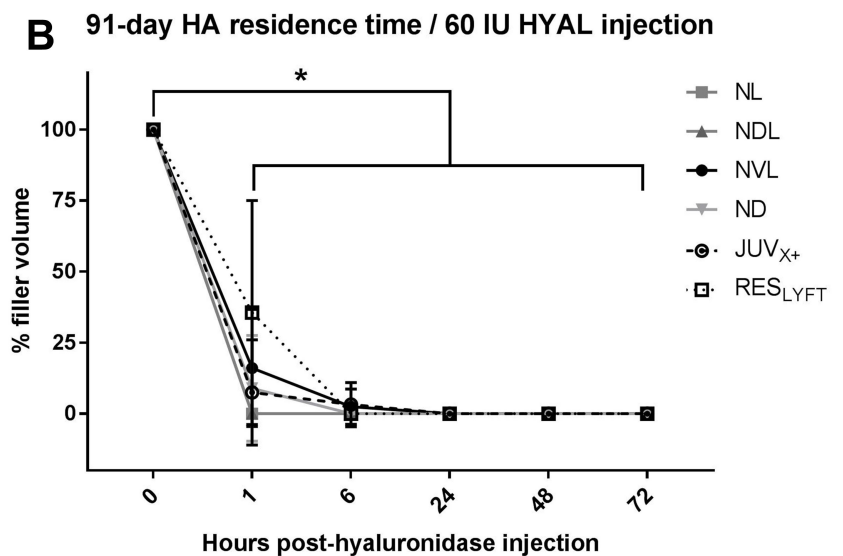

Figure 2 Time course for hyaluronidase-mediated degradation of HA dermal filler implants in hairless mice. Filler volume reduction of subcutaneously implanted HA fillers having 4-day $(\mathbf{A})$ and $9 \mathrm{I}$-day (B) filler residence times and injected with $30 \mathrm{IU}$ or $60 \mathrm{IU}$ hyaluronidase (HYAL) per $0 . \mathrm{I}$-mL HA filler based on surface projection measurements over a 72-h period. Data points with error bars represent mean \pm SD. Asterisks *Indicate significant reduction $(p<0.05)$ in filler volumes between pre- and posthyaluronidase injection. 
Table I Histological Examination to Assess Local Irritation at the Filler Implantation Sites Two Weeks After Hyaluronidase Injection

\begin{tabular}{|c|c|c|c|c|c|c|c|}
\hline \multirow[b]{2}{*}{ Filler Residence Time } & \multicolumn{7}{|c|}{ Histology Scores ${ }^{\dagger}$} \\
\hline & NLL & NL & NDL & NVL & ND & $\mathbf{J U V} \mathbf{V}_{\mathbf{X}+}$ & RES $_{\text {LYFT }}$ \\
\hline 4 days $^{\ddagger}$ & $0.9 \pm 0.7$ & $0.7 \pm 0.6$ & $1.3 \pm 1.1$ & $0.5 \pm 0.6$ & $1.3 \pm 1.1$ & $1.1 \pm 1.0$ & $1.7 \pm 1.0$ \\
\hline 91 days $^{\S}$ & N/A & $0.9 \pm 0.6$ & $1.2 \pm 1.6$ & $1.3 \pm 0.6$ & $2.1 \pm 1.0$ & $2.4 \pm 0.6$ & $2.2 \pm 1.0$ \\
\hline
\end{tabular}

Notes: ${ }^{\dagger}$ Scoring criteria based on ISO $10993-6$ - Test for Local Effects after Implantation; Data expressed as means \pm SD; ${ }^{\ddagger}$ Treated with a single dose of 30 IU hyaluronidase/0.I-mL filler implant; ${ }^{\S}$ Treated with two doses of 30 IU hyaluronidase/0.I-mL filler implant.

Abbreviation: N/A, not applicable.

residence time (Figure 3) and 91-day filler residence time (Figure 4) two weeks after hyaluronidase injection. The histological features and gross examinations at the implantation sites did not reveal any notable signs of irritation two weeks after hyaluronidase injection.

\section{Discussion}

HA-based dermal filler is considered the gold standard for dermal fillers because of its good performance and safety profile. ${ }^{21} \mathrm{HA}$ is structurally homogenous across different living organisms and poses minimal risk in humans. Many of the current HA dermal fillers are manufactured using non-animal derived HA and have low immunogenic profiles. ${ }^{5}$ In addition, chemical modifications to generate crosslinked HA polymers have proven to be an effective method for enhancing the durability of HA fillers against rapid degradation without compromising its biocompatibility. ${ }^{12,22}$ One clear advantage of HA-based dermal fillers over other types is its reversibility via minimally invasive procedure using hyaluronidase. Hyaluronidase injections have been shown to quickly and effectively remove inappropriately implanted HA fillers that could cause severe adverse events, as well as other unwanted outcomes. ${ }^{7,11}$ There are a variety of hyaluronidase formulations approved for human use. These formulations include hyaluronidases derived from purified crude extracts of ovine or testicular tissue, or manufactured using recombinant technology. ${ }^{23}$

For HA dermal fillers, it has been widely regarded that higher HA content, degree of crosslinking and cohesive property confer greater resistance to hyaluronidasemediated degradation. ${ }^{24,25}$ HA filler formulations among the different families of HA filler products vary in cohesive property (monophasic vs biphasic), HA content, and degree of crosslinking, hence the need to establish how effective each type of hyaluronidase is in dissolving these HA fillers. A recent review of available literatures that investigated the in vitro and in vivo degradation of HA fillers has reported that monophasic HA gels as generally more resistant to hyaluronidase-mediated degradation than biphasic HA gels. Also, no consensus was established on which type of hyaluronidase is most superior in dissolving HA fillers. ${ }^{17} \mathrm{~A}$ blind randomized study on the reversal of HA filler injection had demonstrated comparable efficacy between the use of 20 and $40 \mathrm{U}$ ovine hyaluronidase in reducing the volume of $0.2 \mathrm{~mL}$ HA filler bolus implants based on significantly lower palpation scores compared to non-treated and saline-treated HA filler implants. The study also reported that identical treatments of ovine hyaluronidase showed no clinically significant difference in HA filler volume reduction among different filler implants from three major HA filler manufacturers, despite

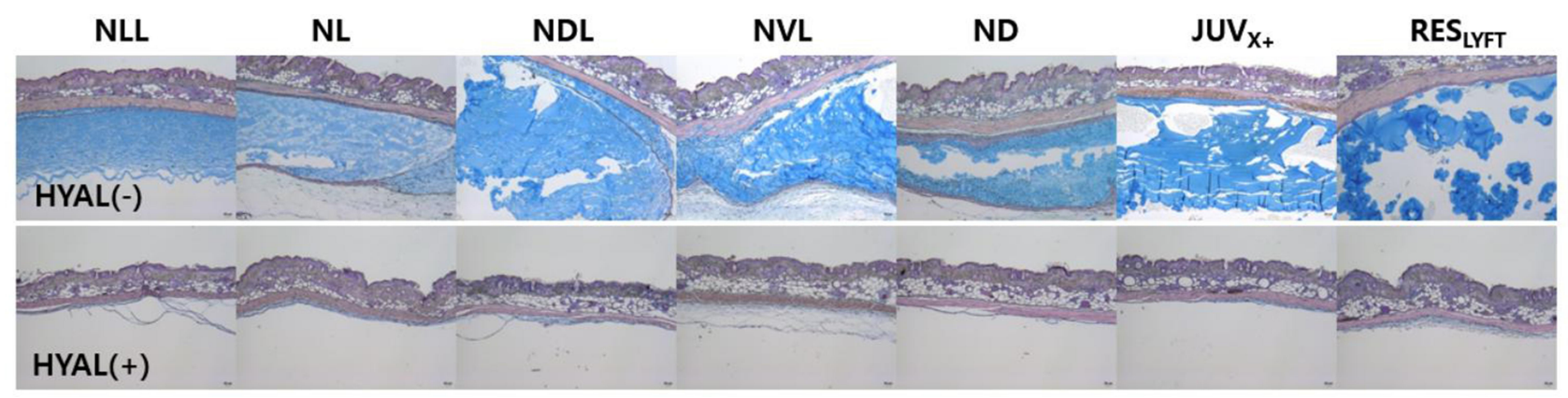

Figure 3 Cross section of sites with subcutaneously implanted HA dermal fillers (4-day filler residence time) before and two weeks after injection with 30 IU hyaluronidase (HYAL) per 0.1-mL HA filler volume. Alcian blue-stained areas show the HA filler layer before HYAL injection (top) and 2 weeks after treatment (bottom). 


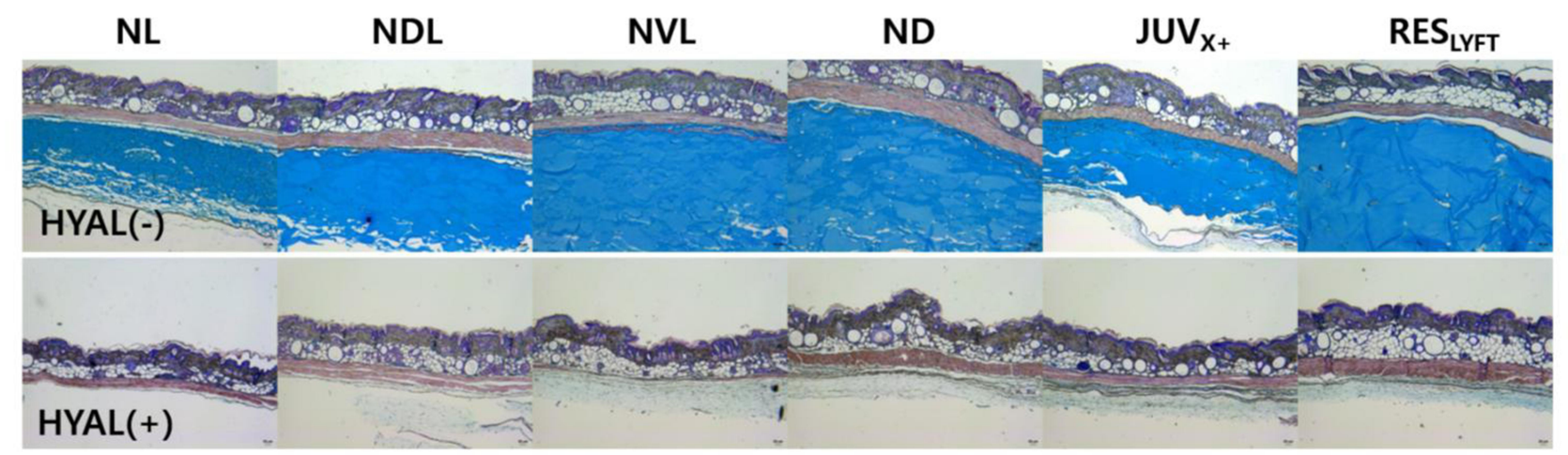

Figure 4 Cross section of sites with subcutaneously implanted HA dermal fillers ( 9 I-day filler residence time) before and two weeks after injection with 60 IU hyaluronidase (HYAL) per 0.I-mL HA filler volume. Alcian blue-stained areas show the HA filler layer before HYAL injection (top) and two weeks after treatment (bottom).

variability in cohesive properties, HA content and degree of crosslinking. ${ }^{26}$

As new formulations of HA dermal fillers are developed, it remains crucial to establish their susceptibility to hyaluronidase-mediated degradation as a safety measure for unforeseen events. In this regard, using in vivo animal models is considered a more reliable system for evaluating hyaluronidase-mediated degradation of HA fillers since the degradation process can be monitored in an environment that closely resembles the clinical setting. Compared to in vitro assays that measure hyaluronidase-mediated degradation under highly controlled conditions, ${ }^{27}$ in vivo models allow for the incorporation of variables that mimic treatment modalities and local tissue environment, such as placement of the hyaluronidase injection relative to the filler location, enzyme diffusion from the injection site, filler swelling and tissue integration, enzyme deactivation and clearance, competing enzyme substrate, inflammatory responses, and other cellular interactions. Factoring these variables provides a more accurate assessment of the susceptibility of an HA dermal filler to hyaluronidase-mediated degradation as well as the efficacy of the hyaluronidase treatment, thereby having more relevance to its clinical application. ${ }^{14,26,28}$

All HA dermal fillers belonging to the NEU series are manufactured as having a monophasic gel phase similar to $\mathrm{JUV}_{\mathrm{X}+}$, and is very distinct from the biphasic gel phase of RES $_{\text {LYFT. }}{ }^{15,19}$ The HA dermal fillers from the NEU series possess the same content of HA, but with varying degree of BDDE crosslinking. Despite differences in the physicochemical properties among the five NEU HA dermal fillers and two comparative HA dermal fillers, the present study demonstrated comparable susceptibility among these different HA-based dermal fillers to hyaluronidase-mediated degradation in vivo. One notable observation was the requisite to increase the hyaluronidase dosage from 30 IU to $60 \mathrm{IU}$ per $0.1-\mathrm{mL}$ HA filler implant when the filler residence time reached 91 days prior to treatment in order to achieve similar rate of HA filler degradation as that of their 4-day filler residence time counterparts. These findings suggest that tissue integration may likely affect the susceptibility of HA dermal fillers to hyaluronidasemediated degradation. Nevertheless, the increase in dosage of hyaluronidase required for efficient degradation of these HA dermal filler implants did not elicit any remarkable adverse events in the surrounding tissues, indicating that the procedure is safe. Further investigation is warranted to determine physical and physiological factors that affect the efficacy of hyaluronidase-mediated degradation. Moreover, a more comprehensive investigation on the in vivo hyaluronidase-mediated degradation of HA fillers from the NEU series and several other HA-based dermal fillers of similar formulation may provide additional insights on the interaction between the HA filler implants and hyaluronidase.

\section{Conclusion}

Hyaluronidase-mediated degradation is one of the main advantages of HA-based dermal fillers over other forms of dermal fillers as it allows for reversibility of the process in the event of inappropriate implantation or occurrence of unwanted adverse events. While most, if not all HA dermal fillers contain crosslinked HA polymers intended to withstand rapid degradation from endogenous hyaluronidase, their composition and chemical modification should not prevent rapid degradation from hyaluronidase treatment when such procedure is necessitated. In this study, the in vivo hyaluronidase-mediated degradation of different HA dermal fillers from the NEU family of products 
and two leading commercial HA filler products, with up to three months filler residence time, showed no significant difference in their susceptibility to exogenous hyaluronidase suggesting that hyaluronidase treatment to reverse soft-tissue augmentation of these HA dermal filler products in the clinical setting is expected to provide similar outcomes. In addition, degradation of HA filler implants with prolonged filler residence time may require a higher dosage of hyaluronidase to achieve similar rate of reversal as newly implanted HA fillers, and is not expected to elicit any serious adverse events at the site of injection.

\section{Acknowledgments}

The study was sponsored by Medytox, Inc.

\section{Disclosure}

S.S. Kwak, K.H. Yoon, J.H. Kwon, W-H Kang, C-H Rhee, G-H Yang and D.J.M. Cruz are employees of Medytox, Inc., a biopharmaceutical company based in South Korea. The authors report no other conflicts of interest in this work.

\section{References}

1. Fraser JR, Laurent TC, Laurent UB. Hyaluronan: its nature, distribution, functions and turnover. J Intern Med. 1997;242(1):27-33. doi:10.1046/j.1365-2796.1997.00170.x

2. Necas J, Bartosikova L, Brauner P, Kolar J. Hyaluronic acid (hyaluronan): a review. Vet Med-Czech. 2008;53(8):397-411. doi:10.17221/ 1930-VETMED

3. Holmstrom B, Ricica J. Production of hyaluronic acid by a streptococcal strain in batch culture. Appl Microbiol. 1967;15 (6):1409-1413. doi:10.1128/AM.15.6.1409-1413.1967

4. Dicker KT, Gurski LA, Pradhan-Bhatt S, Witt RL, Farach-Carson MC, Jia X. Hyaluronan: a simple polysaccharide with diverse biological functions. Acta Biomater. 2014;10(4):1558-1570. doi:10.1016/j. actbio.2013.12.019

5. Kim JE, Sykes JM. Hyaluronic acid fillers: history and overview. Facial Plast Surg. 2011;27(6):523-528. doi:10.1055/s-0031-1298785

6. Beleznay K, Humphrey S, Carruthers JD, Carruthers A. Vascular compromise from soft tissue augmentation: experience with 12 cases and recommendations for optimal outcomes. $J$ Clin Aesthet Dermatol. 2014;7(9):37-43.

7. Landau M. Hyaluronidase caveats in treating filler complications. Dermatol Surg. 2015;41(Suppl 1):S347-S353. doi:10.1097/ DSS.0000000000000555

8. Csoka AB, Frost GI, Stern R. The six hyaluronidase-like genes in the human and mouse genomes. Matrix Biol. 2001;20(8):499-508. doi:10.1016/S0945-053X(01)00172-X

9. Chanmee T, Ontong P, Itano N. Hyaluronan: a modulator of the tumor microenvironment. Cancer Lett. 2016;375(1):20-30. doi:10.1016/j.canlet.2016.02.031

10. Menzinger S, Kaya A, Saurat JH, Kaya G. Injected hyaluronidase reduces the volume of exogenous hyaluronate fillers in mice and results in clinical improvement in a patient with pretibial myxedema. Dermatopathology (Basel). 2016;3(3):61-67. doi:10.1159/000446699
11. Buhren BA, Schrumpf H, Hoff NP, Bolke E, Hilton S, Gerber PA. Hyaluronidase: from clinical applications to molecular and cellular mechanisms. Eur J Med Res. 2016;21:5. doi:10.1186/s40001-0160201-5

12. De Boulle K, Glogau R, Kono T, et al. A review of the metabolism of 1,4-butanediol diglycidyl ether-crosslinked hyaluronic acid dermal fillers. Dermatol Surg. 2013;39(12):1758-1766. doi:10.1111/ dsu. 12301

13. Gold MH. What's New in Fillers in 2010? J Clin Aesthet Dermatol. 2010;3(8):36-45.

14. Shumate GT, Chopra R, Jones D, Messina DJ, Hee CK. In vivo degradation of crosslinked hyaluronic acid fillers by exogenous hyaluronidases. Dermatol Surg. 2018;44(8):1075-1083. doi:10.1097/DSS.0000000000001525

15. Bogdan Allemann I, Baumann L. Hyaluronic acid gel (Juvéderm ${ }^{\mathrm{TM}}$ ) preparations in the treatment of facial wrinkles and folds. Clin Interv Aging. 2008;3(4):629-634. doi:10.2147/CIA.S3118

16. Brandt FS, Cazzaniga A, Brandt F. Hyaluronic acid gel fillers in the management of facial aging. Clin Interv Aging. 2008;3(1):153-159. doi:10.2147/CIA.S2135

17. Paap MK, Silkiss RZ. The interaction between hyaluronidase and hyaluronic acid gel fillers - a review of the literature and comparative analysis. Plastic Aesthet Res. 2020;7:36.

18. Joo HJ, Woo YJ, Kim JE, Kim BJ, Kang H. A randomized clinical trial to evaluate the efficacy and safety of lidocaine-containing monophasic hyaluronic acid filler for nasolabial folds. Plast Reconstr Surg. 2016;137(3):799-808. doi:10.1097/01.prs.0000479965.14775.f0

19. Pak C, Park J, Hong J, Jeong J, Bang S, Heo CY. A phase III, randomized, multi-center, double-masked, matched-pairs, active-controlled trial to compare the efficacy and safety between neuramis deep and restylane in the correction of nasolabial folds. Arch Plast Surg. 2015;42(6):721-728. doi:10.5999/aps.2015.42.6.721

20. ISO 10993-6. Biological Evaluation of Medical Devices. Part 6: Tests for Local Effects After Implantation. International Organization for Standardization; 2016.

21. Betemps JB, Marchetti F, Lim T, et al. Projection capacity assessment of hyaluronic acid fillers. Plast Aesthet Res. 2018;5:19. doi:10.20517/ 2347-9264.2018.24

22. Yeom J, Bhang SH, Kim BS, et al. Effect of cross-linking reagents for hyaluronic acid hydrogel dermal fillers on tissue augmentation and regeneration. Bioconjug Chem. 2010;21(2):240-247. doi:10.1021/bc9002647

23. Dunn AL, Heavner JE, Racz G, Day M. Hyaluronidase: a review of approved formulations, indications and off-label use in chronic pain management. Expert Opin Biol Ther. 2010;10(1):127-131. doi:10.1517/14712590903490382

24. Jones D, Tezel A, Borrel M. In vitro resistance to degradation of hyaluronic acid dermal fillers by ovine testicular hyaluronidase. Dermatol Surg. 2010;36(1):804-809. doi:10.1111/j.1524-4725.20 10.01550.x

25. Sall I, Ferard G. Comparison of the sensitivity of 11 crosslinked hyaluronic acid gels to bovine testis hyaluronidase. Polym Degrad Stabil. 2007;92(5):915-919. doi:10.1016/j.polymdegradstab.20 06.11 .020

26. Juhász ML, Levin MK, Marmur ES. The kinetics of reversible hyaluronic acid filler injection treated with hyaluronidase. Dermatol Surg. 2017;43(6):841-847. doi:10.1097/DSS.0000000000001084

27. Buhren BA, Schrumpf H, Bolke E, Kammers K, Gerber PA. Standardized in vitro analysis of the degradability of hyaluronic acid fillers by hyaluronidase. Eur J Med Res. 2018;23(1):37. doi:10.1186/s40001-018-0334-9

28. Girish KS, Kemparaju K. The magic glue hyaluronan and its eraser hyaluronidase: a biological overview. Life Sci. 2007;80 (21):1921-1943. 


\section{Publish your work in this journal}

Clinical, Cosmetic and Investigational Dermatology is an international, peer-reviewed, open access, online journal that focuses on the latest clinical and experimental research in all aspects of skin disease and cosmetic interventions. This journal is indexed on CAS.
The manuscript management system is completely online and includes a very quick and fair peer-review system, which is all easy to use. Visit http://www.dovepress.com/testimonials.php to read real quotes from published authors. 